\title{
Medidas de prevenção e controle da covid-19: revisão integrativa
}

\author{
Covid-19 prevention and control measures: integrative review
}

Medidas de prevención y control de covid-19: revisión integrativa

Karla Hellen Dias Soares ${ }^{1 *}$, Luana da Silva Oliveira ${ }^{1}$, Renata Karolaine Flor da Silva ${ }^{1}$, Dayanne Caroline de Assis Silva ${ }^{1}$, Ariany Cristine do Nascimento Farias ${ }^{1}$, Estela Maria Leite Meirelles Monteiro ${ }^{1}$, Milton Cezar Compagnon ${ }^{1}$.

\section{RESUMO}

Objetivo: Analisar as evidências científicas disponíveis na literatura sobre as medidas de prevenção e controle da COVID-19. Métodos: Trata-se de uma revisão integrativa de artigos publicados em português, inglês ou espanhol, do ano de 2020, nas bibliotecas/bases Biblioteca Virtual em Saúde (BVS) e Medical Literature Analysis and Retrieval System Online (MEDLINE/Pubmed), sendo a amostra final composta por 08 artigos. A pergunta norteadora foi: Quais as medidas de prevenção e controle da COVID-19? Resultados: A partir da busca de estudos por meio de cruzamentos duplos e triplos, foram encontrados 2.246 artigos, dentre estes, 08 artigos foram selecionados que correspondiam ao tema do estudo, estes foram divididos em duas classes: 1 - A influência ou a percepção dos usuários em relação às medidas de prevenção da COVID-19 a partir das mídias digitais e 2 - As medidas de prevenção e controle da COVID-19 aplicadas pela gestão sanitária. Considerações Finais: Infere-se que, sendo a pandemia desencadeada por uma patologia emergente, ainda com estudos incipientes, concorreu para a disseminação de informações por vezes conflitantes pelos próprios representantes legais, gerando incredulidade e insegurança da população para adesão às medidas sanitárias.

Palavras-chave: Infecções por coronavirus, Pandemias, Síndrome respiratória aguda grave, Prevenção de doenças, Fatores de risco.

\begin{abstract}
Objective: To analyze the scientific evidence available in the literature on the prevention and control measures of COVID-19. Methods: This is an integrative review of articles published in Portuguese, English or Spanish, from the year 2020, in the libraries/databases Virtual Health Library (VHL) and Medical Literature Analysis and Retrieval System Online (MEDLINE/Pubmed), being the final sample consisting of 08 articles. The guiding question was: What are the prevention and control measures of COVID-19? Results: From the search for studies through double and triple crossings, 2,246 articles were found, among these, 08 articles were selected that corresponded to the study theme, these were divided into two classes: 1 - The influence or perception of users in relation to COVID-19 prevention measures from digital media and 2 - COVID-19 prevention and control measures applied by health management. Final Considerations: It appears that, since the pandemic was triggered by an emerging pathology, still with incipient studies, it contributed to the dissemination of sometimes conflicting information by the legal representatives themselves, generating incredulity and insecurity of the population to adhere to health measures.
\end{abstract}

Keywords: Coronavirus infections, Pandemics, Severe acute respiratory syndrome, Disease prevention, Risk factors.

1 Universidade Federal de Pernambuco (UFPE), Recife - PE. “E-mail: karlahellen01@gmail.com 


\section{RESUMEN}

Objetivo: Analizar la evidencia científica disponible en la literatura sobre las medidas de prevención y control de COVID-19. Métodos: Se trata de una revisión integradora de artículos publicados en portugués, inglés o español, a partir del año 2020, en las bibliotecas/bases de datos Biblioteca Virtual en Salud (BVS) y Sistema de Análisis y Recuperación de Literatura Médica en Línea (MEDLINE/Pubmed), siendo la muestra final consta de 08 artículos. La pregunta orientadora fue: ¿Cuáles son las medidas de prevención y control de COVID-19? Resultados: De la búsqueda de estudios a través de cruces dobles y triples se encontraron 2246 artículos, entre estos se seleccionaron 08 artículos que correspondían a la temática de estudio, estos se dividieron en dos clases: 1 - La influencia o percepción de usuarios en relación con las medidas de prevención del COVID19 de los medios digitales y 2 - Medidas de prevención y control del COVID-19 aplicadas por la gestión sanitaria. Consideraciones Finales: Se infiere que, dado que la pandemia fue desencadenada por una patología emergente, aún con estudios incipientes, contribuyó a difusión de información a veces conflictiva por parte de propios representantes legales, generando incredulidad e inseguridad de la población para adherirse las medidas de salud.

Palabras clave: Infecciones por coronavirus, Pandemias, Síndrome respiratorio agudo grave, Prevención de enfermedades, Factores de riesgo.

\section{INTRODUÇÃO}

Em 2019, na cidade de Wuhan, na China, teve origem um surto do novo coronavírus que logo se espalhou mundialmente, caracterizando-se como pandemia no início de março de 2020 pela Organização Mundial da Saúde (OMS) (OPAS, 2020). O SARS-CoV-2 é da família do Betacoronavírus e atua provocando a destruição das paredes alvéolo-capilares levando à uma perda na interface entre o espaço intra-alveolar e o estroma circundante, resultando em vazamento do fluido e preenchimento dos sacos alveolares (KAKODKAR P, et al., 2020). O Ministério da Saúde (2020), descreve a Doença do Coronavírus 2019 (COVID-19), como uma infecção com apresentação clínica semelhante à gripe pelo vírus Influenza e ambas atingem o sistema respiratório.

Os casos da referida patologia variam desde formas assintomáticas até o desenvolvimento de pneumonia grave. Todavia, entre os sintomas mais comuns destacam-se a tosse, pirexia, rinorreia, dor de garganta, anosmia, ageusia, distúrbios gastrintestinais (náuseas/vômitos/diarreia), astenia, hiporexia e dispneia (BRASIL, 2020). No tocante a transmissão viral, esta pode ocorrer por contato, fômites e por gotículas. Por esta razão, técnicas de higiene das mãos e etiquetas respiratórias, ou seja, cuidados ao tossir e/ou espirrar, ajudam a prevenir sua disseminação (KAKODKAR P, et al., 2020).

Nessa perspectiva, Berriós CF, et al. (2020) destaca que até o presente momento, por ser uma doença infectocontagiosa emergente, a adoção das medidas de prevenção da COVID-19 são a melhor opção para o controle da propagação do vírus. Isto posto, com o intuito de conter a transmissão do SARS-CoV-2, fronteiras foram fechadas e diversas recomendações foram estabelecidas, como o isolamento social, orientações a frequente lavagem das mãos, à limpeza e desinfecção de superfícies com mais rigor e o uso obrigatório de máscaras em ambientes públicos.

Como mencionado, a carência de conhecimentos acerca da doença, concorreu para as poucas evidências científicas no tratamento preventivo, culminando na evolução da COVID-19 em populações consideradas de maior suscetibilidade, o grupo de risco, a exemplo de idosos, indivíduos portadores de doenças crônicas e imunodeprimidos (OPAS, 2020; PETROVA D, et al., 2020). Destarte, o tratamento foi centrado nos casos de agravamento da dificuldade respiratória, insuficiência cardíaca, choque séptico e insuficiência renal, culminando em elevada demanda de indivíduos que necessitavam de assistência ventilatória, terapia renal substitutiva, cuidados especializados e outros suportes avançados de vida em Unidade de Terapia Intensiva (UTI) (SAHU KK, et al., 2020).

Em contrapartida, a necessidade de retorno com segurança às atividades laborais e ao convívio social até que se tenha acesso a vacinação, vem requerer um processo de aprendizado pela população para estabelecer medidas estruturais na preparação do ambiente e no emprego de técnicas de etiquetas respiratórias (PENNA GO, et al., 2020). 
Assim, a adesão dos indivíduos a tais medidas requer um processo de conscientização, mediado por uma relação de confiança destes com os representantes dos órgãos de saúde. A ausência deste processo concorre para situações de insegurança da população quanto a eficiência das medidas preventivas, e consequentemente dificulta a adesão dessas, diante da instabilidade nas orientações disseminadas (LIMA LSC, et al., 2020).

Por conseguinte, a adoção de medidas de prevenção, desenvolvidas mediante embasamento científico, pode contribuir para a contenção viral e consequente redução de casos evitando colapso no sistema de saúde. Por essa razão, se faz necessário investigar quais as evidências científicas disponíveis acerca das medidas de prevenção e controle da COVID-19 a fim de ensiná-las e implementá-las nos diversos espaços sociais.

Logo, o presente estudo teve como objetivo analisar as evidências científicas disponíveis na literatura sobre as medidas de prevenção e controle da COVID-19.

\section{MÉTODOS}

Trata-se de uma revisão integrativa, a qual permite a elaboração de uma síntese do conhecimento já relatado na literatura sobre uma determinada temática e promove uma análise de ampla compreensão dos dados encontrados (KAKUSHI LE e ÉVORA YDM, 2016). Para assegurar o rigor na condução do estudo, foram realizadas as seguintes etapas: Identificação da temática e do problema de pesquisa; Definição dos critérios de inclusão e exclusão; Identificação dos estudos selecionados; Categorização dos estudos selecionados; Análise e interpretação dos resultados e síntese do conhecimento evidenciado nos artigos analisados ou apresentação da revisão (BOTELHO LLR, et al., 2011).

No presente estudo, foi formulada a seguinte pergunta norteadora: Quais as medidas de prevenção e controle da COVID-19? Com a finalidade de responder esta pergunta, a coleta de dados ocorreu nos meses de maio a julho de 2020 por dois juízes independentes, nas seguintes bibliotecas/bases de dados: BVS e MEDLINE/Pubmed. Para a busca dos artigos utilizou-se os Descritores em Ciências da Saúde - DeCS/MeSH e suas respectivas traduções: Infecções por Coronavirus/Coronavirus Infections/Infecciones por Coronavirus; Pandemias/Pandemics/Pandemias; Síndrome Respiratória Aguda Grave/Severe Acute Respiratory Syndrome/Síndrome Respiratorio Agudo Grave; Prevenção de Doenças/Disease Prevention/Prevención de Enfermedades; Fatores de Risco/Risk Factors/Factores de Riesgo. Foram realizados cruzamentos duplos e triplos e combinados com o operador booleano AND.

Como critérios de inclusão, foram definidos artigos originais, publicados, nos idiomas português, inglês e espanhol, que abranjam as medidas de prevenção e controle da COVID-19 e que respondam a pergunta norteadora da revisão. Os critérios de exclusão estabelecidos foram: teses, dissertações, editoriais, capítulos de livros, carta ao leitor, revisões da literatura, comentário, comunicação livre, artigos duplicados, estudos de perspectiva, diretrizes, relatos de experiência e fuga do objetivo.

O nível de evidência foi avaliado de acordo com Stleter CB, et al. (1998), com a seguinte categorização: nível I - Estudos de metanálise de múltiplos estudos controlados e randomizados; nível II - Estudos individuais com desenho experimental; nível III - Estudos quase-experimentais, séries temporais ou caso-controle; nível IV - Estudos descritivos (não experimentais ou abordagem qualitativa); nível V - Relatos de caso ou de experiência; nível VI - Opiniões de comitês de especialistas, incluindo interpretações de informações não baseadas em pesquisas, opiniões reguladoras ou legais. Todos os artigos selecionados foram categorizados com o nível de evidência IV.

A partir da busca de estudos por meio dos cruzamentos duplos e triplos, foram encontrados 2.246 artigos nas bases de dados utilizadas. Conforme o seguimento das etapas de desenvolvimento da presente revisão, 2.008 artigos foram excluídos de acordo com a leitura dos títulos e 159 artigos excluídos após a leitura dos resumos por não estarem em consonância com a temática ou por não responderem à questão norteadora. Dos 79 artigos restantes, procedeu-se com a leitura dos estudos na íntegra, restando assim, 08 artigos que demonstraram afinidade com o tema proposto no presente estudo (Figura 1). 
Figura 1 - Fluxograma do processo de seleção dos estudos, adaptado do Preferred Reporting Items for Systematic Review and Meta-Analyses (PRISMA).

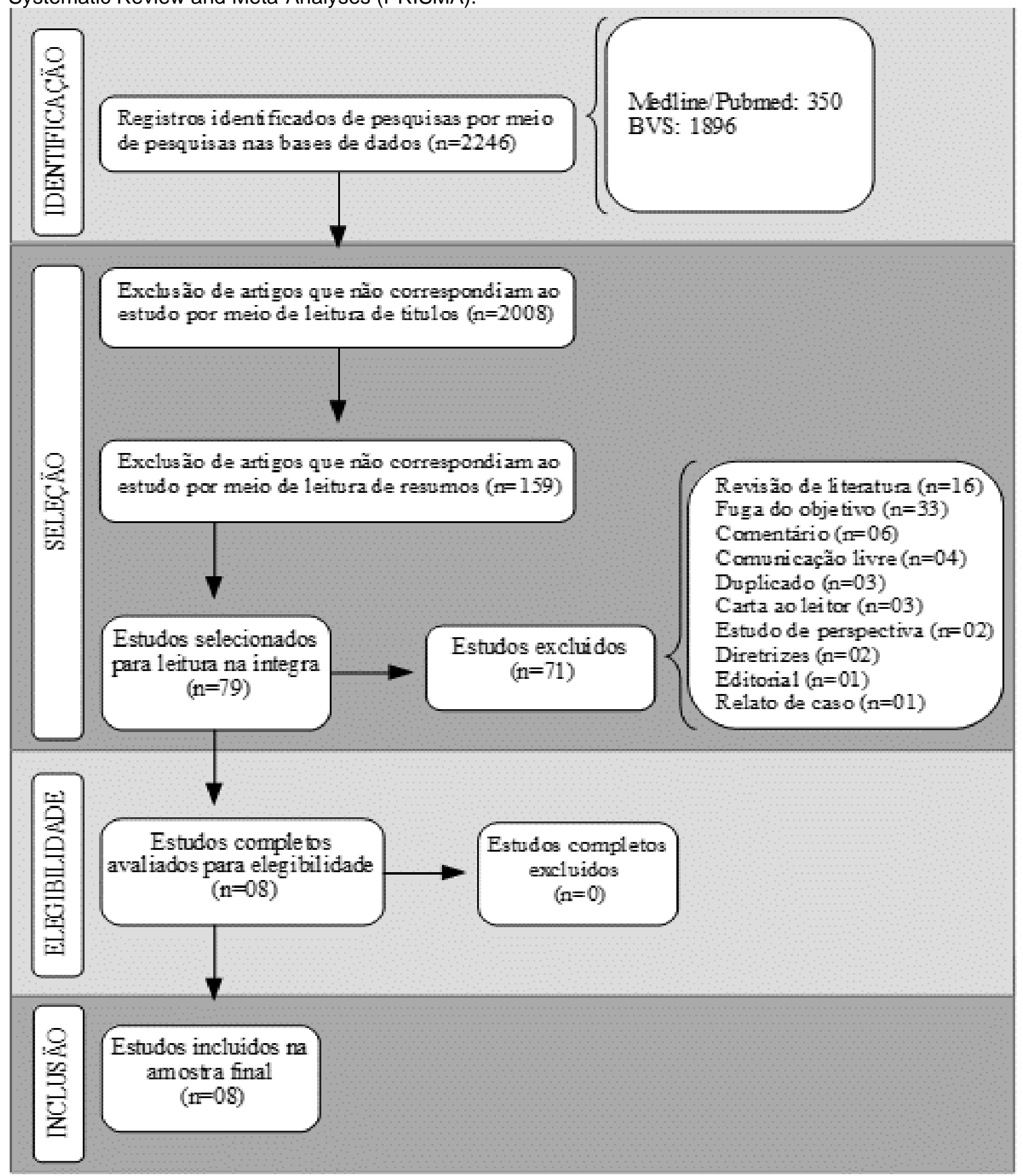

Fonte: Soares KHD, et al., 2021. Adaptado de Preferred Reporting Items for Systematic Review and MetaAnalyses (PRISMA), 2020.

\section{RESULTADOS}

Dos 8 estudos recuperados, todos apresentaram nível de evidência IV, denotando estudos descritivos quantitativos e qualitativos. Todos os estudos foram desenvolvidos no ano de 2020 e apresentaram-se no idioma inglês. No que se refere ao local do estudo, dois foram desenvolvidos nos Estados Unidos da América, três na China, um na Finlândia, um no Catar e um na Itália (Quadro 1). 
Quadro 1 - Descrição dos estudos selecionados para revisão segundo título do artigo, autores, país, ano de publicação, objetivo e resultados.

\begin{tabular}{|c|c|c|c|}
\hline Artigo/Autores & País/Ano & Objetivo & Resultados \\
\hline $\begin{array}{l}\text { 1. Preventive Behaviors } \\
\text { Conveyed on YouTube to } \\
\text { Mitigate Transmission of } \\
\text { COVID-19: Cross-Sectional } \\
\text { Study (BASCH, et al., 2020). }\end{array}$ & $\begin{array}{l}\text { Estados } \\
\text { Unidos da } \\
\text { América/ } \\
2020\end{array}$ & $\begin{array}{l}\text { Investigar o conteúdo de vídeos do } \\
\text { YouTube relacionados a COVID-19. }\end{array}$ & $\begin{array}{l}\text { O Centro de Controle e Prevenção de Doenças dos EUA lista os seguintes } \\
\text { comportamentos de prevenção como sendo os principais: higienização das } \\
\text { mãos; evitar contato com pessoas enfermas; ficar em casa quando doente; } \\
\text { cobrir a tosse ou espirro com lenço de papel e depois descartá-lo; usar } \\
\text { máscara facial ao cuidar de alguém que está doente e/ou quando estiver } \\
\text { doente; e limpar e desinfetar objetos e superfícies. Ao analisar os } 100 \text { vídeos } \\
\text { mais acessados do youtube utilizando "coronavírus" como "palavra-chave" } \\
\text { constatou-se que menos de um terço dos vídeos abordava qualquer um dos } \\
\text { sete principais comportamentos de prevenção e que a maioria menciona } \\
\text { morte ou sugere medo ou ansiedade. }\end{array}$ \\
\hline $\begin{array}{l}\text { 2. Understanding coronavirus } \\
\text { disease (COVID-19) risk } \\
\text { perceptions among the public } \\
\text { to enhance risk } \\
\text { communication efforts: a } \\
\text { practical approach for } \\
\text { outbreaks, Finland, February } \\
2020 \text { (LOHINIVA, et al., } \\
\text { 2020). }\end{array}$ & $\begin{array}{l}\text { Finlândia/ } \\
2020\end{array}$ & $\begin{array}{c}\text { Apreender a percepção de risco e a } \\
\text { confiança em relação às } \\
\text { autoridades públicas no contexto } \\
\text { da doença por coronavírus, através } \\
\text { da análise de emails e mensagens } \\
\text { de mídia social. }\end{array}$ & $\begin{array}{l}\text { Os resultados mostraram que havia uma falta de crença de que uma pessoa } \\
\text { pode controlar individualmente a propagação da epidemia e, em vez disso, } \\
\text { havia uma forte crença de que as autoridades podem fazer isso. As } \\
\text { recomendações de comunicação de risco incluíram enfatizar o que os } \\
\text { indivíduos podem fazer para evitar a propagação da infecção, como higiene } \\
\text { das mãos, etiqueta para tosse e evitar tocar olhos, nariz e boca. }\end{array}$ \\
\hline $\begin{array}{l}\text { 3. Corona Virus (COVID-19) } \\
\text { "Infodemic" and Emerging } \\
\text { Issues through a Data Lens: } \\
\text { The Case of China (HUA, et } \\
\text { al., 2020). }\end{array}$ & China/ 2020 & $\begin{array}{l}\text { Analisar a linha do tempo, baseado } \\
\text { principalmente em jornais chineses, } \\
\text { mídias sociais e outros dados da } \\
\text { plataforma digital das principais } \\
\text { ações realizadas pelo governo e } \\
\text { pelo povo ao longo de três meses. }\end{array}$ & $\begin{array}{l}\text { Constatou que, embora houvesse um atraso inicial na resposta, uma } \\
\text { combinação única de forte governança, regulamentação estrita, forte } \\
\text { vigilância comunitária, participação dos cidadãos, uso inteligente de } \\
\text { tecnologias digitais, foram alguns dos fatores-chave nos esforços da China } \\
\text { para combater este vírus. Ao considerar o Coronavírus um desastre invisível } \\
\text { e não mensurável, é crucial ter informações corretas e oportunas para } \\
\text { interromper sua disseminação, bem como na promoção de medidas } \\
\text { preventivas dessa doença. }\end{array}$ \\
\hline $\begin{array}{l}\text { 4. Application of refined } \\
\text { management in the } \\
\text { prevention and control of } \\
\text { coronavirus disease } 2019 \\
\text { epidemic in non-isolated } \\
\text { areas of a general hospital. } \\
\text { (XU, et al., 2020). }\end{array}$ & China/ 2020 & $\begin{array}{c}\text { Relatar a experiência na prevenção } \\
\text { e controle da epidemia de doença } \\
\text { por coronavírus } 2019 \text { (COVID-19) } \\
\text { em áreas não isoladas } \\
\text { (ambulatórios, enfermarias e área } \\
\text { administrativa) de um hospital } \\
\text { geral. }\end{array}$ & $\begin{array}{l}\text { Em } 31 \text { de janeiro de } 2020 \text {, não havia infecção por COVID-19 adquirida no } \\
\text { hospital entre os funcionários deste, confirmando a eficácia das estratégias } \\
\text { de controle e prevenção da COVID-19 proposta pelos órgãos de gestão em } \\
\text { saúde. As principais condutas foram: aferição de temperatura, higienização } \\
\text { das mãos, uso de máscara, exposição de cartazes informativos e uso de } \\
\text { equipamentos de proteção individual pelos profissionais de saúde. }\end{array}$ \\
\hline
\end{tabular}




\begin{tabular}{|c|c|c|c|}
\hline Artigo/Autores & País/Ano & Objetivo & Resultados \\
\hline $\begin{array}{l}\text { 5. Mental health problems } \\
\text { and social media exposure } \\
\text { during COVID-19 outbreak. } \\
\text { (GAO, et al., 2020). }\end{array}$ & China/ 2020 & $\begin{array}{c}\text { Descrever a prevalência e } \\
\text { distribuição de dois principais } \\
\text { transtornos mentais: ansiedade e } \\
\text { depressão entre a população } \\
\text { chinesa, relacionando-os com a } \\
\text { exposição a mídias sociais durante } \\
\text { a epidemia da COVID-19. }\end{array}$ & $\begin{array}{l}\text { A prevalência de depressão, ansiedade e combinação de depressão e } \\
\text { ansiedade foi de } 48,3 \%, 22,6 \% \text { e } 19,4 \% \text { respectivamente durante o período } \\
\text { COVID-19 em Wuhan, China. Mais de } 80 \% \text { dos participantes relataram estar } \\
\text { frequentemente expostos às mídias sociais. Após o controle de covariáveis, } \\
\text { os indivíduos que estavam frequentemente expostos às mídias sociais foram } \\
\text { associados positivamente a altas chances de ansiedade e depressão } \\
\text { associada a ansiedade em comparação com os menos expostos às mídias } \\
\text { sociais. }\end{array}$ \\
\hline $\begin{array}{l}\text { 6. Top Concerns of Tweeters } \\
\text { During the COVID-19 } \\
\text { Pandemic: Infoveillance } \\
\text { Study (ABD-ALRAZAQ, et al., } \\
\text { 2020). }\end{array}$ & Catar/ 2020 & $\begin{array}{l}\text { Identificar os principais tópicos } \\
\text { publicados pelos usuários do } \\
\text { Twitter relacionados à pandemia da } \\
\text { COVID-19. }\end{array}$ & $\begin{array}{l}\text { A análise identificou } 12 \text { tópicos, que foram agrupados em quatro temas } \\
\text { principais: origem do vírus; suas fontes; seu impacto nas pessoas, nos } \\
\text { países e na economia; e formas de mitigar o risco de infecção. Essas } \\
\text { comunicações interpessoais indicam que as pessoas se relacionam com o } \\
\text { tópico da COVID-19 no Twitter e que os usuários desta rede social estão } \\
\text { interessados em notificar ou alertar seus amigos e seguidores sobre a } \\
\text { doença. No entanto, o uso sensacionalista do Twitter pode ser um grande } \\
\text { desafio para os esforços de saúde pública e resposta a surtos devido à } \\
\text { disseminação generalizada de teorias da conspiração e da desinformação. }\end{array}$ \\
\hline $\begin{array}{l}\text { 7. Protecting healthcare } \\
\text { workers from SARS-CoV-2 } \\
\text { infection: practical } \\
\text { indications. (FERIOLI, et al., } \\
\text { 2020). }\end{array}$ & Itália/ 2020 & $\begin{array}{c}\text { Fornecer recomendações baseadas } \\
\text { em evidências para o uso correto } \\
\text { de "dispositivos respiratórios" na } \\
\text { emergência COVID-19 e proteger } \\
\text { os profissionais de saúde de } \\
\text { contrair a infecção por SARS-CoV- } \\
2 .\end{array}$ & $\begin{array}{l}\text { O artigo recomenda as seguintes medidas preventivas para pacientes, } \\
\text { profissionais de saúde e comunidade: Lavagem das mãos frequentemente; } \\
\text { Evitar o contato com os olhos, nariz e boca; Espirrar e tossir em um cotovelo } \\
\text { ou lenço dobrado e, em seguida, descartá-lo; Usar máscara cirúrgica na } \\
\text { presença de sintomas respiratórios; Manter uma distância de pelo menos } 1 \mathrm{~m} \\
\text { de pacientes com sintomas respiratórios. }\end{array}$ \\
\hline $\begin{array}{l}\text { 8. Preliminary Estimates of } \\
\text { the Prevalence of Selected } \\
\text { Underlying Health Conditions } \\
\text { Among Patients with } \\
\text { Coronavirus Disease } 2019 \text { - } \\
\text { United States, February 12- } \\
\text { March 28, 2020 (CHOW, et } \\
\text { al., 2020). }\end{array}$ & $\begin{array}{l}\text { Estados } \\
\text { Unidos da } \\
\text { América/ } \\
2020\end{array}$ & $\begin{array}{l}\text { Avaliar condições de risco } \\
\text { relacionadas a comorbidades pré- } \\
\text { existentes, quanto ao } \\
\text { desenvolvimento a forma mais } \\
\text { grave da COVID-19. }\end{array}$ & $\begin{array}{l}\text { Pessoas com doenças pré-existentes devem ter os seguintes cuidados: lavar } \\
\text { as mãos; limpeza e desinfecção de superfícies de alto toque; e o } \\
\text { distanciamento social, incluindo ficar em casa, evitar multidões, reuniões e } \\
\text { viagens e evitar o contato com pessoas doentes. Manter um suprimento de } \\
\text { pelo menos } 30 \text { dias de medicação, um suprimento de } 2 \text { semanas de } \\
\text { alimentos e outras necessidades e conhecimento dos sintomas de COVID- } \\
19 \text { são recomendados para aqueles com condições de saúde subjacentes. } \\
\text { Todas as pessoas devem tomar medidas para se proteger da COVID-19 e } \\
\text { para proteger os outros. Todas as pessoas que estão doentes devem ficar } \\
\text { em casa, exceto para obter cuidados médicos; não deve ir trabalhar; e deve } \\
\text { ficar em isolamento social. }\end{array}$ \\
\hline
\end{tabular}

Fonte: Soares KHD, et al., 2020. 


\section{DISCUSSÃO}

A discussão dos achados dos estudos será apresentada considerando a composição das seguintes categorias: 1 - A influência ou a percepção dos usuários em relação às medidas de prevenção da COVID-19 a partir das mídias digitais e 2 - As medidas de prevenção e controle da COVID-19 aplicadas pela gestão sanitária.

A categoria 1 é composta pelos artigos 1, 2, 3, 5 e 6, que reportam o uso de mídias e plataformas digitais com elevado alcance e interação entre os internautas, como o YouTube e o Twitter (VASCONCELLOS-SILVA PR e CASTIEL LD, 2020). Os demais estudos desta categoria abordam a comunicação virtual destacando as mensagens propagadas mediante as tecnologias e mídias sociais, incluindo a televisão.

No cenário pandêmico, muitos internautas e telespectadores se apropriam desses recursos digitais para difundir e/ou adquirir informações. Entretanto, diante do alcance que a internet tem, tais informações podem ser alteradas, ganhando um teor sensacionalista e promotor de ansiedade na população, fato este que é bem destacado nos artigos 1 e 6 , que analisam essa temática, e trazem como resultados dados que revelam que a exposição prolongada às mídias sociais torna o internauta mais vulnerável à conteúdos sugestivos de morte, ansiedade, medo e depressão com relação à realidade atual. Uma vez que, a maior parte dos conteúdos divulgados sobre a COVID-19 em mídias sociais trazem uma perspectiva negativa, com ênfase em números de óbitos, e não de sobreviventes; fakenews e influências contra as medidas preventivas diante da instabilidade nas orientações propagadas pelas organizações de saúde.

O Artigo 1 analisou 100 dos vídeos mais assistidos no YouTube, utilizando a palavra-chave "coronavírus", e o resultado revela que menos de um terço dos vídeos abordava qualquer um dos sete principais comportamentos de prevenção definidos pelo Centro de Controle de Doenças. A utilização das mídias sociais e plataformas digitais foi discutida no estudo 6 destacando seus benefícios e malefícios, com ênfase no discernimento crítico do internauta para averiguar a veracidade das informações. Diante disso, o Ministério da Saúde do Brasil, disponibilizou em seu site oficial um aplicativo de mensagens no combate às Fake News, o que se constitui uma estratégia de enfrentamento a desinformação (BASCH CH, et al., 2020).

Durante a pandemia, diferentes campanhas de conscientização surgiram, as quais exploravam a importância da lavagem das mãos, da etiqueta respiratória, dentre outras, citadas também nos artigos 1,2, 3, 4, 7 e 8; mas uma ganhou muito destaque, sendo incluída nas propagandas televisivas e nas redes sociais, uma frase que dizia: "Fique em casa", relacionada à estratégia das autoridades civis e sanitárias quanto ao isolamento social, como medida de desacelerar a transmissão do SARS-CoV-2. Assim, prevaleceu uma orientação para que as pessoas doentes se mantivessem isoladas, e as pessoas do grupo de risco evitassem a exposição, como também os cidadãos de forma geral, evitassem sair de casa, limitando seu deslocamento apenas para situações essenciais (BASCH CH, et al., 2020; LOHINIVA AL, et al., 2020; HUA J e SHAW R, 2020; XU C, et al.,2020; FERIOLI M, et al.,2020; CHOW N, et al., 2020).

$\mathrm{O}$ isolamento social, já tem sido avaliado e estudos demonstram que de fato, tal medida contribui para o retardo da transmissão da doença, contudo, o entendimento de ficar em casa, pode motivar alguns infectados a demorarem na busca por assistência médica, procurando os serviços de saúde, apenas quando apresentam sintomas mais graves como a dispneia (MATRAJT L e LEUNG T, 2020). Esta realidade está relacionada ao despreparo inicial dos serviços de saúde em disporem de leitos e de recursos necessários para o diagnóstico e tratamento adequado em um contexto de pandemia, contribuindo assim para o aumento no número de pessoas com as formas mais graves da COVID-19, sem assistência em sua totalidade (NORONHA KVMS, et al., 2020).

Por outro lado, manter-se em casa, o fechamento de estabelecimentos comerciais, paralisação de obras, diminuição nas frotas de ônibus e a abertura exclusiva dos serviços considerados essenciais, contribuiu também para dificuldades econômicas no país, pois, não tendo como manter os salários, com as portas fechadas, muitos empresários dispensaram seus funcionários, enquanto que outros negócios faliram (JOHNSON MT, et al., 2020). Assim, atender as recomendações prolongadas de quarentena ficaram cada vez mais insustentáveis para muitas famílias carentes, pela necessidade de sair diariamente para granjear 0 alimento diário por meio de trabalhos informais (CHRISTOFFEL MM, et al., 2020). 
Vale ressaltar, que o isolamento social é improvável para muitas famílias que dividem pouco espaço com muitas pessoas da sua constituição familiar; outras não têm acesso a água potável para a adequada higiene das mãos e outras condições primárias como saneamento básico, concorrendo para deliberar uma resistência social às orientações de saúde (BONG CL, et al., 2020; RIBEIRO F e LEIST A, 2020).

Logo, podemos inferir que a pandemia vem intensificando alguns agravos sociais nas populações carentes, em vulnerabilidade social, a exemplo do desemprego. Orientações com maior embasamento científico de combate a infecção e prevenção de novos indivíduos acometidos, estabelece que, é necessário isolar casos confirmados ou suspeitos com a doença leve em casa, identificar e acompanhar os contatos, desinfetar o ambiente e usar equipamentos de proteção individual. Além disso, é essencial uma boa ventilação e iluminação natural do ambiente (ADHIKARI SP, et al., 2020; SINGHAL T, 2020).

Outrossim, como forma de atenuar os impactos sociais e econômicos, o governo autorizou a reabertura do comércio com suas adaptações, e disponibilizou para os trabalhadores informais e sem outra renda/auxílio/benefício, um auxílio chamado emergencial para atender algumas das necessidades primárias da população (BRASIL, 2020). Ficou evidenciado no entendimento da saúde como resultante da produção social, que os prejuízos à economia do país desencadeados com a situação de pandemia ampliam os desafios para promoção da saúde principalmente da população com baixo poder aquisitivo.

Diante das situações de vulnerabilidade em saúde, vivenciadas pela população, emerge considerar as consequências na saúde mental, frente às medidas de isolamento social e da divulgação nos meios de comunicação midiático do número crescente de registro diário de mortes por COVID-19 no Brasil e no mundo (RIBEIRO CJN, et al., 2020).

A percepção holística da saúde, concorreu para o desenvolvimento de investigações, que abordou os acometimentos da saúde mental da população durante a situação de pandemia, como verificado no estudo 5 , que as pessoas mais expostas às mídias sociais apresentavam maior risco para ansiedade e depressão (GAO Q, et al., 2020).

Quanto a categoria 2, esta é composta pelos artigos 4, 7 e 8, que reportam as medidas de prevenção e controle da COVID-19 aplicadas pela gestão sanitária. O Artigo 4 reporta as medidas a serem empregadas pelos profissionais no ambiente hospitalar, enquanto o Artigo 7 relaciona os principais cuidados preventivos para pacientes, profissionais de saúde e comunidade; e por último, o Artigo 8 retrata os cuidados às pessoas com comorbidades de saúde (XU C, et al., 2020; FERIOLI M, et al., 2020; CHOW N, et al., 2020).

Os três artigos recomendam a lavagem das mãos como medida de higiene em combate a disseminação do vírus, o artigo 7 acrescenta o uso de soluções à base de álcool ( $>65 \%)$, quando as mãos não estiverem visivelmente sujas, e alerta para higiene das mãos sempre que houver contato com secreções respiratórias (FERIOLI M, et al., 2020).

O uso de soluções alcoólicas para higienização das mãos e superfícies é antigo e se tornou amplamente utilizado devido a facilidade de aplicação, porém é recomendado apenas em locais que não possuem acesso a pia e água potável e/ou quando as mãos estão visivelmente limpas, sua eficácia depende da concentração e tipo do álcool, do volume aplicado e tempo de contato (SEQUINEL R, et al., 2020).

O uso de máscara foi citado nos artigos da seguinte forma: o Artigo 4 direciona o uso para os profissionais da saúde, o Artigo 7, além dos profissionais, estende para as pessoas com sintomas respiratórios e por último, o Artigo 8 não cita o uso de máscaras (XU C, et al., 2020; FERIOLI M, et al., 2020; CHOW N, et al., 2020).

Tais achados divergem das atuais orientações de saúde definidas e divulgadas pelos órgãos oficiais, como a Agência Nacional de Vigilância Sanitária (ANVISA), que lançou um documento com orientações gerais para a população, não profissional da saúde, sobre o uso de máscara, no qual orienta que os indivíduos utilizem máscaras de fabricação doméstica/artesanal de tecido, quando precisar sair de casa, orienta até mesmo a quantidade mínima de cinco máscaras de uso individual para cada pessoa, e o uso de máscara cirúrgica destinado a profissionais da saúde, cuidadores e indivíduos infectados pelo Coronavírus (ANVISA, 2020). 
Isto posto, se faz necessário atentar também para o uso e reuso correto da máscara artesanal, observando as condições de higiene e conservação da mesma, assim como o uso e descarte adequado da máscara industrial, uma vez que a precaução é imprescindível tanto para os profissionais de saúde, quanto para a população em geral, constituindo-se determinantes para o não contágio pelo SARS-CoV-2 (NETO ARS e FREITAS DRJ, 2020).

Ao considerar que os profissionais da saúde apresentam maior risco de se infectar, faz-se necessário assegurar todas as medidas de proteção, para poderem atuar com segurança na continuidade do cuidado e impossibilitar que novos pacientes sejam acometidos pelo COVID-19 (SINGHAL T, 2020).

Destarte, a ANVISA estabeleceu a Nota Técnica 05/2020, que traz orientações específicas para os profissionais da assistência em saúde, recomendando o uso de máscara cirúrgica quando em contato com pacientes confirmados e suspeitos de infecção pelo SARS-CoV-2, e uso de máscara do tipo N-95/PFF2 durante procedimentos que geram aerossolização (BRASIL, 2020).

Essa Nota Técnica também informa a importância e os cuidados durante o uso e a retirada dos equipamentos de proteção individual (EPI's). Como forma de impor a população as medidas de prevenção contra a COVID-19, foi sancionada a Lei oㅜ 14.019, de 2 de julho de 2020, que estabelece a obrigatoriedade do uso de máscara em locais públicos e privados acessíveis ao público e em locais fechados em que haja agrupamento de pessoas (BRASIL, 2020).

Outro ponto de destaque nas medidas sanitárias é o distanciamento de pelo menos um metro entre as pessoas, conforme o Artigo 7, recomendação que está de acordo com a folha informativa da OPAS/OMS, 2020 (FERIOLI M, et al., 2020).

O Artigo 8, informa que as pessoas consideradas do grupo de risco para a COVID-19, ou seja, pessoas que possuem problemas de saúde subjacentes, devem priorizar como medida de prevenção, se manter em casa, exceto para obter cuidados médicos, sendo recomendado ainda manter um suprimento de pelo menos trinta dias de medicação, e um suprimento de duas semanas de alimentos (CHOW N, et al.,2020).

A preocupação com esta população, que possui alguma comorbidade pré-existente, decorre da elevada ocorrência de casos mais graves da doença do Coronavírus, requerendo internamentos em UTI (GAO Q, et al., 2020). Emerge desse modo, como referido no artigo 5, a importância do isolamento social, evitando multidões, reuniões, viagens e o contato com pessoas infectadas (GAO J, et al., 2020).

Estudos explorando medidas de prevenção e controle começaram a aumentar gradualmente. Investigações científicas para fornecer maneiras válidas e confiáveis de gerenciar esse tipo de emergência de saúde pública tanto no curto quanto no longo prazo são necessárias para minimizar o impacto do surto. As agências governamentais incorporaram rapidamente as descobertas científicas recentes às políticas públicas da comunidade para desacelerar e/ou prevenir a disseminação da COVID-19 (ADHIKARI SP, et al., 2020).

Ademais, no que se refere à limitação do presente estudo, observou-se uma carência de pesquisas acerca da temática em questão. Contudo, evidencia-se a necessidade de investir em estudos voltados ao desenvolvimento de medidas de prevenção e contenção do SARS-CoV-2, principalmente no cenário brasileiro, uma vez que evidências a respeito do tema estavam ausentes na literatura nacional.

\section{CONSIDERAÇÕES FINAIS}

O fato da pandemia ser desencadeada por uma patologia emergente, ainda com estudos incipientes, concorreu para a disseminação de informações por vezes conflitantes pelos próprios representantes legais, gerando incredulidade e insegurança da população para adesão às medidas sanitárias. Nessa perspectiva, a enfermagem pode contribuir com estratégias e orientações que promovem a utilização das medidas sanitárias no controle de doenças infectocontagiosas, assim como, é capaz de transmitir maior segurança e equilíbrio emocional à população. Visto que, foi destacado uma elevada associação no desenvolvimento de ansiedade e depressão em indivíduos que estavam frequentemente expostos às mídias sociais. Outrossim, entre as medidas sanitárias recomendadas, destacaram-se: higienização das mãos, uso de álcool gel e uso de máscara. Para mais, o elevado número de casos graves e de óbitos, culminou com a recomendação ao isolamento social por parte das autoridades sanitárias. 


\section{REFERÊNCIAS}

1. ABD-ALRAZAQ A, et al. Top Concerns of Tweeters During the COVID-19 Pandemic: Infoveillance Study. J Med Internet Res, 2020; 22(4): e19016.

2. ADHIKARI SP, et al. Epidemiology, causes, clinical manifestation and diagnosis, prevention and control of coronavirus disease (COVID-19) during the early outbreak period: a scoping review. Infect Dis Poverty, 2020; 9(29): 01-12.

3. AGÊNCIA NACIONAL DE VIGILÂNCIA SANITÁRIA. Nota Técnica GVIMS/GGTES/ANVISA N 04/2020. Orientações para Serviços de Saúde: Medidas de Prevenção e Controle que devem ser adotadas durante a assistência aos casos suspeitos ou confirmados de infecção pelo novo Coronavírus (SARS-CoV-2). Brasília: 31 de Março de 2020. Disponível em: https://www20.anvisa.gov.br/segurancadopaciente/index.php/alertas/item/nota-tecnica-n-04-2020gvims-ggtes-anvisa-atualizada. Acesso em: 8 out. 2020.

4. AGÊNCIA NACIONAL DE VIGILÂNCIA SANITÁRIA. Orientações Gerais - Máscaras faciais de uso não professional. Brasília: 03 de Abril de 2020. Disponível em: https://agenciabrasilia.df.gov.br/wp-conteudo/uploads/2020/04/NTM\%C3\%A1scaras-Tecido-Anvisa.pdf-2.pdf. Acesso em: 8 out. 2020.

5. BASCH CH, et al. Preventive Behaviors Conveyed on YouTube to Mitigate Transmission of COVID-19: Cross-Sectional Study. JMIR Public Health Surveill, 2020; 6(2): e18807.

6. BERRÍOS CF, et al. Prevención y medidas de protección frente a la infección por SARS-CoV-2. Neumol Pediatr, 2020; 15 (2): $308-316$.

7. BONG CL, et al. The COVID-19 Pandemic: Effects on Low- and Middle-Income Countries. Anesth Analg, 2020; 131(1): 86-92.

8. BOTELHO LLR, et al. O MÉTODO DA REVISÃO INTEGRATIVA NOS ESTUDOS ORGANIZACIONAIS. Gestão E Sociedade, 2011; 5(11): 121-136.

9. BRASIL. Diário Oficial da União. Lei $\mathrm{N}^{\circ}$ 13.982, de 2 de abril de 2020. Disponível em: https://www.in.gov.br/en/web/dou/-/lei-n-13.982-de-2-de-abril-de-2020-250915958. Acesso em: 9 out. 2020.

10. BRASIL. Diário Oficial da União. Lei $\mathrm{N}^{\circ} 14.019$, de 2 de julho de 2020. Disponível em: https://www.in.gov.br/en/web/dou/-/lei-n-14.019-de-2-de-julho-de-2020-264918074. Acesso em: 9 out. 2020.

11. CHOW N, et al. Preliminary Estimates of the Prevalence of Selected Underlying Health Conditions Among Patients with Coronavirus Disease 2019 - United States, February 12-March 28, 2020. MMWR Morb Mortal Wkly Rep, 2020; 69(13): 382-386.

12. CHRISTOFFEL MM, et al. A (in) visibilidade da criança em vulnerabilidade social e o impacto do novo coronavírus (COVID19). Rev. Bras. Enferm. [online], 2020; 73(2): e20200302.

13. FERIOLI M, et al. Protecting healthcare workers from SARS-CoV-2 infection: practical indications. Eur Respir Ver, 2020: 29(155): 200068.

14. GAO J, et al. Mental health problems and social media exposure during COVID-19 outbreak. PLoS ONE, 2020; 15(4): e0231924.

15. GAO Q, et al. The epidemiological characteristics of 2019 novel coronavirus diseases (COVID-19) in Jingmen, Hubei, China. MedRxiv preprint, 2020; 69(13): 381-386.

16. HUA J, SHAW R. Corona Virus (COVID-19) "Infodemic" and Emerging Issues through a Data Lens: The Case of China. Int. J. Environ. Res. Public Health, 2020; 17(7): 01-12.

17. JOHNSON MT, et al. Mitigating social and economic sources of trauma: The need for universal basic income during the coronavirus pandemic. Psychol Trauma, 2020; 12(1): 191-192.

18. KAKODKAR $P$, et al. A Comprehensive Literature Review on the Clinical Presentation, and Management of the Pandemic Coronavirus Disease 2019 (COVID-19). Cureus, 2020; 12(4): e7560.

19. KAKUSHI LE, ÉVORA YDM. As redes sociais na educação em enfermagem: revisão integrativa da literatura. Rev. Latino-Am. Enfermagem, 2016; 24: e2709.

20. LIMA LSC, et al. Reflections on biosafety in the context of COVID-19: repercussions for professionals and for the population. Research, Society and Development, 2020; 9(9): e818997993.

21. LOHINIVA AL, et al. Understanding coronavirus disease (COVID-19) risk perceptions among the public to enhance risk communication efforts: a practical approach for outbreaks, Finland, February 2020. Euro Surveill, 2020; 25(13): 2000317.

22. MATRAJT L, LEUNG T. Evaluating the Effectiveness of Social Distancing Interventions to Delay or Flatten the Epidemic Curve of Coronavirus Disease. Emerg Infect Dis, 2020; 26(8): 1740-1748.

23. BRASIL. Ministério da Saúde. Sobre a doença: O que é Covid-19. Disponível em: https://coronavirus.saude.gov.br/sobre-a-doenca\#o-que-é-covid. Acesso em: 7 set. 2020.

24. BRASIL. Ministério da Saúde. Fake News. Disponível em: https://www.saude.gov.br/fakenews. Acesso em: 15 set. 2020.

25. MOHER D, et al. Preferred reporting items for systematic reviews and meta-analyses: the PRISMA statement. PLoS Med, 2009; 6(7): e1000097. 
26. NETO ARS, FREITAS DRJ. Utilização de máscaras: indicações de uso e manejo durante a pandemia de Covid-19. Cogitare enferm, 2020; 25: e72867.

27. NORONHA KVMS, et al. Pandemia por COVID-19 no Brasil: análise da demanda e da oferta de leitos hospitalares e equipamentos de ventilação assistida segundo diferentes cenários. Cad. Saúde Pública [online], 2020; 36(6): e00115320.

28. OPAS/BRASIL. Folha informativa COVID-19 - Escritório da OPAS e da OMS no Brasil. Disponível em: https://www.paho.org/pt/covid19. Acesso em: 7 set. 2020.

29. PENNA GO, et al. PNAD COVID-19: um novo e poderoso instrumento para Vigilância em Saúde no Brasil. Ciênc. saúde coletiva [online], 2020; 25(9): 3567-3571.

30. PETROVA D, et al. La obesidad como factor de riesgo en personas con COVID-19: posibles mecanismos e implicaciones. Aten Primaria, 2020; 52(7): 496-500.

31. RIBEIRO CJN, et al. Intervenções de restrição de mobilidade social durante a pandemia de COVID-19 e suas repercussões psicossociais no Brasil. Enferm. Foco, 2020;11(1,n.esp): 179-181.

32. RIBEIRO R, LEIST A. Who is going to pay the price of Covid-19? Reflections about an unequal Brazil. Int $J$ Equity Health, $2020 ; 19(1): 91$.

33. SAHU KK, et al. COVID-2019: update on epidemiology, disease spread and management. Monaldi Archives for Chest Disease, 2020; 90(1).

34. SEQUINEL R, et al. Soluções à base de álcool para higienização das mãos e superfícies na prevenção da covid-19: compêndio informativo sob o ponto de vista da química envolvida. Química Nova, 2020; 43(5): 679-684.

35. SINGHAL T. A Review of Coronavirus Disease-2019 (COVID-19). Indian J Pediatr. 2020; 87(4): 281-286.

36. STETLER CB, et al. Evidence-based practice and the role of nursing leadership. J Nurs Adm, 1998; 28(7-8):45-53.

37. VASCONCELLOS-SILVA PR, CASTIEL LD. COVID-19: as fakenews e o sono da razão comunicativa gerando monstros: a narrativa dos riscos e os riscos das narrativas. Cad. Saúde Pública (Online), 2020; 36(7): e00101920.

38. XU C, et al. Application of refined management in the prevention and control of coronavirus disease 2019 epidemic in non-isolated areas of a general hospital. Int J Nurs Sci, 2020; 7(2): 143-147. 